\title{
Digital Model of Surface Equipment within the Digital Oilfield Framework
}

\author{
Iakov S. Korovin* and Maxim G. Tkachenko \\ Southern Federal University, Rostov-on-Don \\ *Corresponding author
}

\begin{abstract}
In the paper we describe a new approach to the construction of a model, applied for a complex for the extraction and processing of hydrocarbon raw materials. The basis of the model is a group of independently functioning components, organized on the basis of modern Arificial Intelligence methods, including various neural network solutions. The integration of the modules is carried out by introducing into the model of the digital oilfield the concept of energy flows transmitted between the simulated components. We propose to consider the joint transfer and transformation of several independent types of energy, including electrical energy, fluid energy, and also mass-volume transfer values by each independent block. While applying components of neural network structures, the values of the input and output energy fluxes must complement the vector quantities characterizing the internal parameters of the model.
\end{abstract}

Keywords-Digital oilfield, energy flow, reservoir modeling, neural networks.

\section{INTRODUCTION}

During the process of planning the construction of oil production equipment, there is often a need to determine the mutual influence of the modified nodes on the operating components of the surface infrastructure of the field. A similar situation arises at the initial stages of development of new deposits, when it is required to estimate the material costs for the construction of complexes for the extraction, preparation and trans portation of petroleum products.

At present, the so-called Nennton iterations are used to solve the modeling problem, which, on the basis of the balance of pressures and mass at the network nodes, reduces the problemto solving a systemof linear equations. In this paper, we propose a new method based on the use of a directory of pre-trained tunable neural network models, united by the concept of energy transfer between the components of the modeled deposit.

As a tool for modeling the relationships between model components, it is proposed to use approaches to the analysis of graph models [1], describing the relationship between the tuned neural network components of the field infrastructure.

\section{DIGIT AL MODEL OF SURFACE EQUIPMENT}

While conducting our research there arose the need to modify the models of the structural components of the oil collection and treatment complex proposed in earlier works [2]. Thus, the combination of all composite models within the framework of a single field model led to the need to introduce a universal interface of interaction between simulated subsystems, including booster pumping station (BPS) models, water conduit networks, production and injection wells.

Interface below depicted is proposed to introduce the energy component into the model of each block. In each of the component models, the energy component is represented as a vector, the elements of which characterize the values of the transmitted energy of a different nature. We proposed to supplement the models described earlier with vectors characterizing the following types of transmitted energy:

- $\quad$ electric energy used by models of pumping units to increase the energy components of transmitted liquid media;

- the energy of the fluid (potential and kinetic) obtained by the medium from the formation or models of pumping equipment and irretrievably lost during transmission through models of pipelines and bush equipment;

- $\quad$ "Energy" of volume-mass transfer, characterizing in the form of normalized numerical values, depending on the initial settings, the volume or mass of the object being transported by the model of the working medium (water or oil products).

Taking into account the expansion of the previously proposed models of the components of the dynamic model of the field by energy links, the combined two-level hierarchical model of the field will include the following interrelated models at the first (upper) level:

- collection network model;

- $\quad$ BPS model;

- model of pipe network for maintenance of reservoir pressure (MRP);

- $\quad$ injection well model;

- $\quad$ FWKO (Free Water Knock-out) model.

Schematically, this model is shown in Fig. 1. 


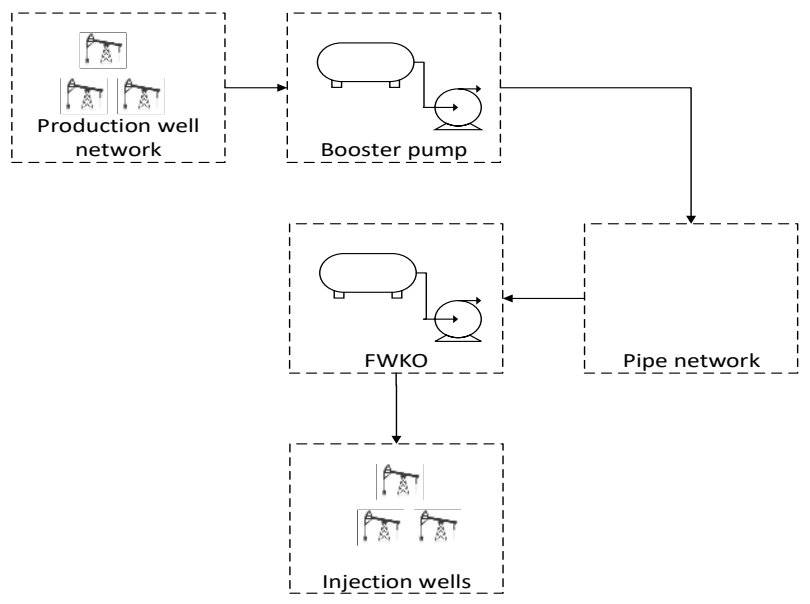

FIGURE I. UPPER LEVEL OF DYNAMICAL OILFIELD MODEL.

In this model, the transmission networks of electric power are not shown, since for the model test it was not possible to obtain data on losses in transmission networks from production plants. If the modeling of losses in the transmission networks of electrical energy is refused, all the energy-dependent components of the upper-level model (collection network, BPS, FWKO) are conditionally combined, and the electric power transmission lines in the simulation correspond to the structure of the tubing network of the MRP.

\section{COMPONENT MODELS}

At the second level of the hierarchical model, the main blocks of the upper level are divided into composite elements, up to the models of pumping units used in oil collection and treatment operations.

In this paper, the collection network model is a set of coupled modified well models, including the models of a production tree, a tubing and a pump unit (Fig. 2).
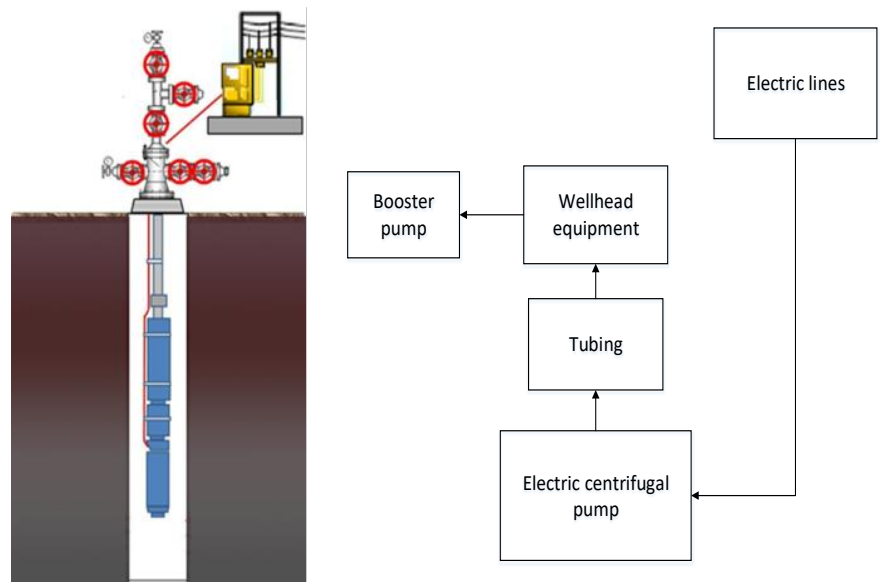

FIGURE II. SCHEME AND A MODEL OF OIL PRODUCTION WELL.

The model of an injection well with the combined reservoir model is not divided into composite components, it is assumed that this component has a similar structure to the production well model, however, there are no active components (ESP and electric power transmission lines).
The FWKO model is schematically shown in Figure 3. The model includes:

- $\quad$ point of communication with the network of wells;

- $\quad$ separators;

- $\quad$ small BPS;

- $\quad$ point of connection with the tubing network of MRP.

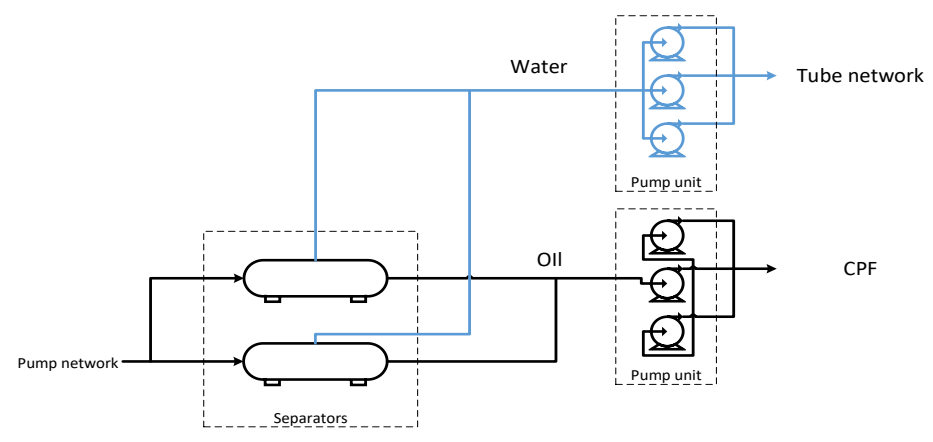

FIGURE III. FWKO MODEL.

During the simulation, the BPSs are not splitted up into separate component blocks, as it was not possible to obtain the technological data for this model from the manufacturing plant. In the future, BPS blocks are considered as single nodes schematically shown in Fig. 4, which ensure the transformation of previously described types of energy flows.

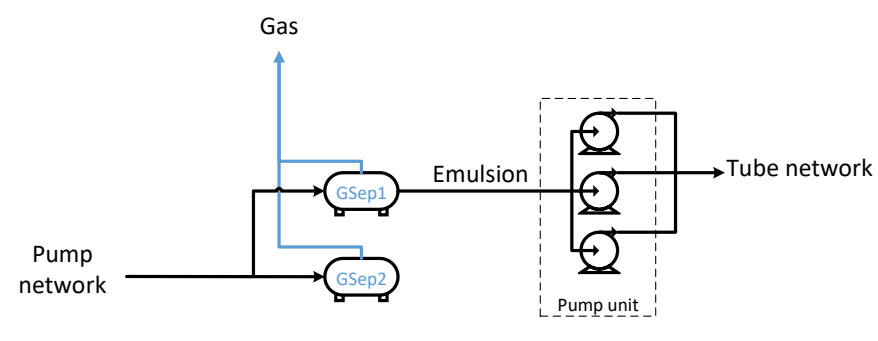

FIGURE IV. BPS MODEL.

Thus, the proposed integrated mathematical model of the technological process includes:

The model of the production well (well cluster model), based on the use of the neural network methodology, establishes the relationship between the number of stages, the gas factor of the pump, the pump rotation speed and the volume of the fluid produced by the well. Also, the model takes into account

- the efficiency of the ESP, the presence of a gas separator at the inlet to the pump;

- a neural network model of conduits, which takes as input the parameters of the water conduit (the diameter of the pipes used, the flow rate of the fluid, the pressure in the pipe, the viscosity and other characteris tics of the fluid being trans ported). The model is trained in such a way as to emit losses of kinetic energy of the fluid that arise in waterways during the transportation of liquid. It is assumed in the work that during 
transmission through conduits the liquid does not change in volume and mass;

- the model of oil gathering networks uses the same data mining tools as the water conduit model, however, when learning the model, the parameters are selected in such a way that they describe the behavior of the pipelines when transporting viscous media (in particular, the data used for training are characterized by a significant decrease in pressure with increasing length pipeline, lead to the formation of a model of higher estimates of kinetic energy losses during transportation of the medium through the oil gathering network model in comparison with model of water conduits). The injection well model establishes the relationship between the wellhead pressure and the volume of fluid injected into the formation

- the model of power networks - is integrated with the model of water conduits, while the electric energy transmitted by the model corresponds to the flows of the model of energy networks, and the energy and volumetric characteristics of the fluid - the model of water conduits.

At the output, the model evaluates the performance of the pump to detect emergencies, the volumetric characteris tics of the transported environment, and the parameters of the pump's energy impact on the complex field model. A detailed description of methods for constructing neural network models for each component can be found in [1], one of the methods for combining a group of models is given in [2].

The proposed management concept for the integrated field model is based on the management principle by regulating the energy flows of the fishery.

In accordance with the proposed approach to managing the field based on the account of energy flows, it is possible to formulate a general algorithm for estimating and predicting the performance of a digital deposit as follows:

1. Forming a library of pre-trained neural network components to form a local estimate and predict the parameters of the component model;

2. Construction of the technological scheme of the oil production complex in the form of a graph whose vertices correspond to the trained components of the model, and the edges to the connections between these components;

3. Simulation of the processes of energy transfer on the constructed graph in order to determine the predictive estimates of the effect of the model parameters on the obtained data on the efficiency of oil product extraction in the field.

This algorithm is shown in Figure 5 below.

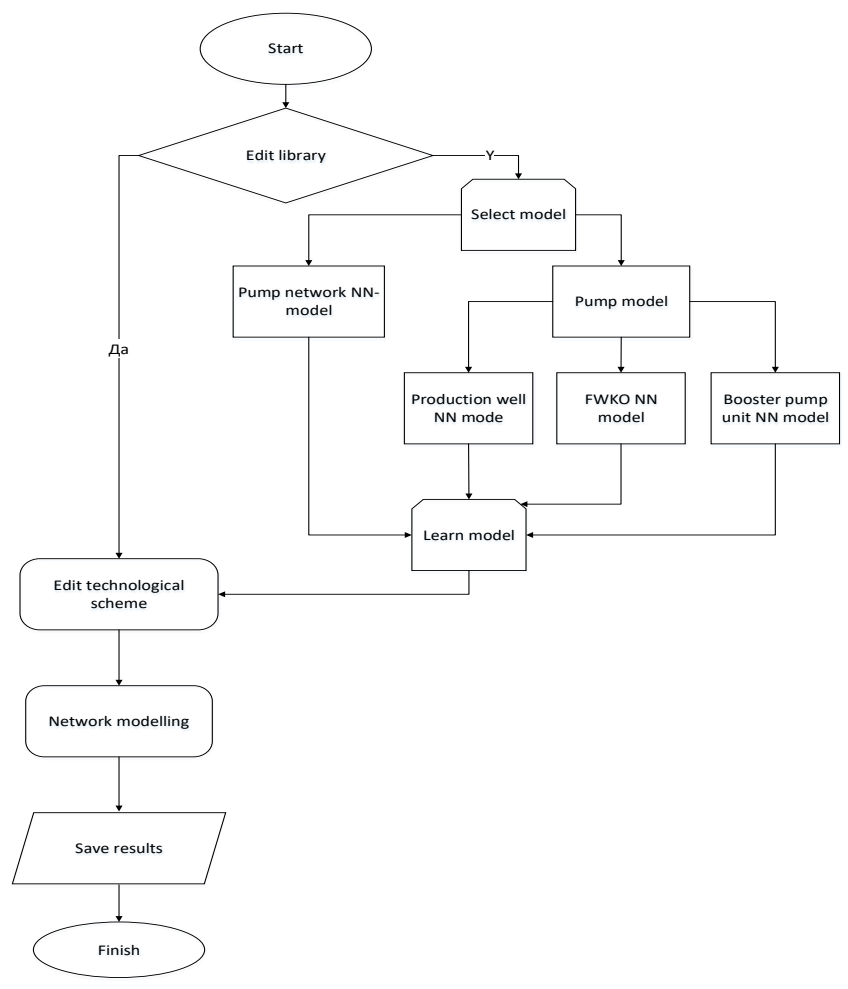

FIGURE V. BPS MODEL.

Figure 5. Block diagram of the algorithm for estimating and predicting the parameters of the digital deposit operation.

All the considered model components in the proposed field model are represented by neural network modules that take as input data the internal parameters of the operation characteristic for each modeled block, as well as additional values of the energy impact values on the modeled component from the external component-related model nodes.

The output data of the neural network model are the parameters of the internal state of the objects (malfunctions, operating modes, etc.) considered in [1], as well as the values of the components of the energy interaction vector of the component (output energy components) transformed as a result of the component's functioning.

Thus, neural network models are tuned to the initial data, describing the properties of energy losses in the elements of the system, and on the properties of energy sources that provide fluid pumping.

To reduce the nomenclature of the simulated nodes, a single neural network model of a centrifugal pump is used to analyze and predict the state of both the ESP of production wells and the centrifugal pumping units of the BPS and FWKO.

The neural network model of the material balance controls (separators installed in the system, change the material balance of fluid motion, since part of the flow (water or gas) is separated from the main one and is no longer taken into account in the model).

The paper proposes to use data on the values of the proposed energy parameters of the model elements that can be directly 
measured on test objects or calculated on the basis of known mathematical relationships for conducting training procedures for neural network models that emit the work of each component in the library of components of the modeled deposit.

The model of each object included in the structure of the generalized model of the field should, in addition to the internal parameters of the operation that determine the state of the control object, also include analys is of the energy component data. Since neural network models are used to model the nodes, the practical approach to realizing the method of estimating the energy parameters of the model is to supplement the vectors of the input and output data with the values of the energy components.

Such a solution makes it possible to evaluate both the internal parameters of the work of each element of the model (according to the structure of the parameters proposed at the previous stages of work) and the external parameters of the model characterizing its energy interaction with other elements of the model.An analys is of the totality of external influences of each of the model elements on the remaining elements can be modeled by constructing a directed graph, whose vertices are directly modeled elements, and the edges are the links between the elements. A detailed description of this method can be found in [1]. In this approach, each of the edges of the graph is marked by the energy interaction vector of the model nodes in this way, any output energy value of the node of one element of the model becomes the input energy component of the other element. In addition, the graphical representation of the model involves the introduction of several terminal nodes that are sources and sinks of energy, these elements include the source of in-plate energy, a source of electrical energy; The drain of energy is the nodes of commercial delivery of oil products and injection wells. The analysis of the interrelation of components in the graph representation of the problem makes it possible to form predictive estimates of the effect of the initial model parameters on the energy components characterizing the performance of the simulated complex.It is assumed that for the tasks simulated in the work, the initial graph of the technological process can be represented in the form of a tree structure in which the directions of the flows of transported media are uniquely determined when the model is formed.

\section{CONCLUSIONS}

The proposed model is a set of intellectual approaches to the modeling of individual objects of the field, united by a common model of the field, described by a graph of connections between the components used froma pre-trained directory. This approach does not allow to form a single intellectual model of the entire deposit, because a wide range of pos sible structural solutions for the entire field can not be represented as a single vector scheme describing both the composition of the complex and the parameters of its components, which is a necessary solution when using neural network approaches. However, the proposed approach, which takes into account the energy balance of the field, uses the intellectual methods proposed in the previous stages of work in modeling each of the components of the integrated model.In the proposed approach, a directed graph describing the structure of the deposit is used to determine the balance of the energy accounted for in the field. To determine this balance, the energy components of the graph should be calculated taking into account the direction of the fluid flows. To perform this calculation, all the relationships of the graph model are replaced by a system of linear equations that include the values of the input and output energy components for each type of energy separately. The solution of the resulting system of equations can be obtained using any known method for solving systems of linear algebraic equations. The proposed model extends the magnitude of the initial energy impact (the pressure at the well exit) through the base components, each of which besides the internal parameters has the ability to transfer and obtain the values of the energy components.

\section{ACKNOWLEDGMENT}

This paper is published due the financial support of the Russian Science Foundation (RSF) via the grant No 15-1900196.

\section{REFERENCES}

[1] Ya.S. Korovin., M.G. Tkachenko, S.V. Kononov, Oilfield equipment's state diagnostics on the basis of data mining technologies (In Russ.), Neftyanoekhozyaystvo= Oil Industry, no. 9, pp. 116-118,2012.

[2] Ya.S. Korovin., M.G. Tkachenko, Method of calculation of coordinates of height of products in system of contactless definition of raznovysotnosti tvs of the active zone of the reactor(In Russ.), Izvestiya Yuzhnogo Federal'nogo Universiteta. Tekhnicheskie nauki = Izvestiya SFedU. Engineering sciences, V. 113, no. 12, pp. 172-178, 2010.

[3] Ya.S. Korovin., M.V. Khisamutdinov, M.G. Tkachenko, Forecasting of oilfield equipment work conditions with the application of evolutionary algorithms and artificial neural networks (In Russ.), Neftyanoe khozyaystvo = Oil Industry, no. 12, pp. 128-133, 2013.

[4] Iakov S. Korovin, Igor A. Kalyaev, Modern Decision Support Systems in Oil Industry: Types, Approaches and Applications, International Conference on Test, Measurement and Comput ational Methods (TMCM) 2015, Chiang Mai, THAILAND; Advances in Computer Science Research. ISBN 978-94-6252-132-2. ISSN 2352-538x, pp. 141-144.

[5] Iakov S. Korovin, The Importance of New Approaches Development and their Implementation in the Oil and Gas Industry in Russian Federat ion-the Current Situation Analysis, International Conference on Advances in Energy, Environment and Chemical Engineering (AEECE) 2015, Changsha, PEOPLES R CHINA; Advances in En gineering Research. ISBN: 978-94-6252-109-4. ISSN: 2352-5401. Atlantis Press, Volume 23, 2015, p.94-97.

[6] Iakov S. Korovin, Anatoly I. Kalyaev, Maksim V. Khisamutdinov, Data Mining Methods Application to the Problem of Handling Corporative Dat aset on Heavy Oil Production, International Conference on Intelligent Control and Computer Application (ICCA), Zhengzhou, PEOPLES R CHINA: JAN 16-17, 2016 Proceedings of the 2016 international conference on intelligent control and computer application, ACSRAdvances in Comptuer Science Research Volume 30, pp. 387-389.

[7] I.S. Korovin, M.V. Khisamutdinov, G. Schaefer, A. Kaly aev, Real-time diagnostics of oil production equipment using data mining, 2016 th International Conference on Informatics, Electronics and Vision (ICIEV), 2016, pp. 1196-1172, DOI: 10.1109/ICIEV.2016. 7760184.

[8] I.S. Korovin, M.V. Khisamutdinov, G. Schaefer, A. Kalyaev, Application of hybrid data mining methods to increase profitability of heavy oil production, 20165th International Conference on Informatics, Electronics and Vision (ICIEV), 2016, pp. 1149 - 1152, DOI: 10.1109/ICIEV.2016.77601 